\title{
DESIGN A WEARABLE DEVICE FOR BLOOD OXYGEN CONCENTRATION AND TEMPORAL HEART BEAT RATE
}

\author{
Cho Zin Myint, Nader Barsoum, Wong Kïng Ing \\ Curtin University of Technology, Sarawak, Malaysia \\ thamiecho@gmail.com, nader.b@curtin.edu.my, wong.kiing.ing@curtin.edu.my
}

\begin{abstract}
The wireless network technology is increasingly important in healthcare as a result of the aging population and the tendency to acquire chronic disease such as heart attack, high blood pressure amongst the elderly. A wireless sensor network system that has the capability to monitor physiological sign such as $\mathrm{SpO}_{2}$ (Saturation of Arterial Oxygen) and heart beat rate in real-time from the human's body is highlighted in this study. This research is to design a prototype sensor network hardware, which consists of microcontroller PIC18F series and transceiver unit. The sensor is corporate into a wearable body sensor network which is small in size and easy to use. The sensor allows a non invasive, real time method to provide information regarding the health of the body. This enables a more efficient and economical means for managing the health care of the population.
\end{abstract}

Keywords: healthcare, wearable devise, body sensor microcontroller, MPLAB IDE

\section{Introduction}

Increase in the demand for aging services is resulted by the increasing elderly population. In recent years, the electronic and wireless network technologies have been improved and its use has progressively benefited in health care for its potential advantages. [1,2]. Recent developments of technology are anticipated to support healthcare professionals effectively and economically in long-term care conveniences. This may reduce the cost of healthcare and enlarge the worth of life of the patient while decreasing the trouble of the professional's healthcare [3]. One significant technology which would benefit from a wireless implementation is an oximeter. As a consequence of small enough to be wearable, less expensive compared to the old health monitoring devices, simple to use, needs no user calibration and accurate enough for clinical use, the pulse oximetry is introduced to the health care. For these reasons, in almost every hospital critical care units and surgical theaters, the pulse oximeter is acknowledged as standard monitoring device [4].

The medical applications of oximeter include monitoring patients during anesthesia, intensive care, or those with conditions such as asthma [5]. This system is advantageous for health status of elderly for a number of reasons. The failure of oxygen delivery to the heart or brain can result the death. Monitoring of oxygen delivery is vital and pulse oximetry measures arterial oxygen saturation $\left(\mathrm{SpO}_{2}\right)$ [6]. Another benefit of pulse oximetry is the capability to measure other critical physiological information from a single compact sensor [7]. Pulse oximetry became accepted for a non-invasive method and it can give immediate data of the arterial oxygen saturation in the patient's blood.

The technology of pulse oximetry is based upon the different red and infrared light absorption individualities of oxygenated hemoglobin $\left(\mathrm{HbO}_{2}\right)$ and deoxygenated hemoglobin $(\mathrm{Hb})$. The heart beat rate can be detected by the measurements of both electrocardiography and blood pressure. A red, and infrared LEDs and a photodiode are used to transmit red and infrared light source to human tissue and collect the transmitted light source respectively. Transmittance pulse oximetry offers several significant advantages over reflectance pulse oximetry, including higher signal amplitude (and thus higher signal-to-noise ratio), more convenient access, and less pressure dependence [8].

A sensor node is run under Assembly Language which is a one- to- one correspondence between the instructions of assembly language and machine language, it is fairly straightforward to translate instructions from the assembly language to machine language. Using Assembly Language program is to have direct control over the system hardware [9].

This paper describes the hardware implementation to detect the saturation of oxygen in the blood and the pulse rate in real time. The software or firmware for the embedded system of pulse oximeter is written in Assembly language, compiled and programmed into PIC18LF452 Microcontroller using Microchip MPLAB IDE (Integrated Development Environment) and PICkit2 programmer. The experimental results of pulse oximeter are also included in this paper. Figure 1 shows the implementation of pulse oximeter, and the schematic diagram of IR/Red driver is given in figure 2. 


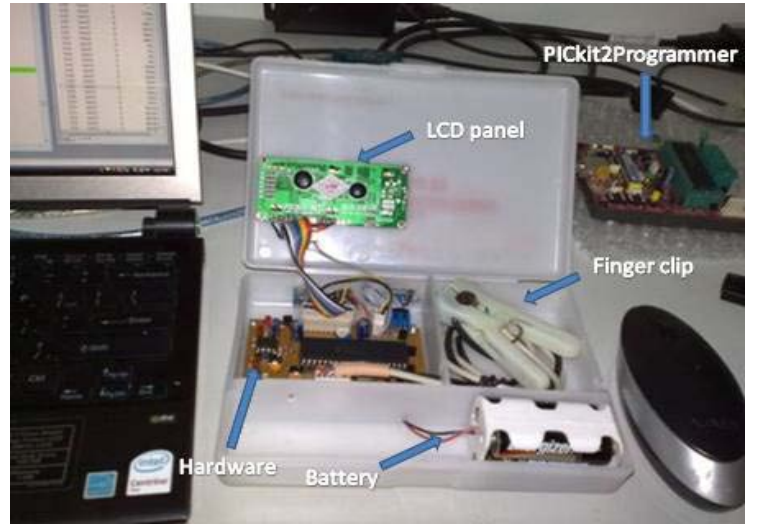

Figure 1: Experimental set-up of pulse oximeter

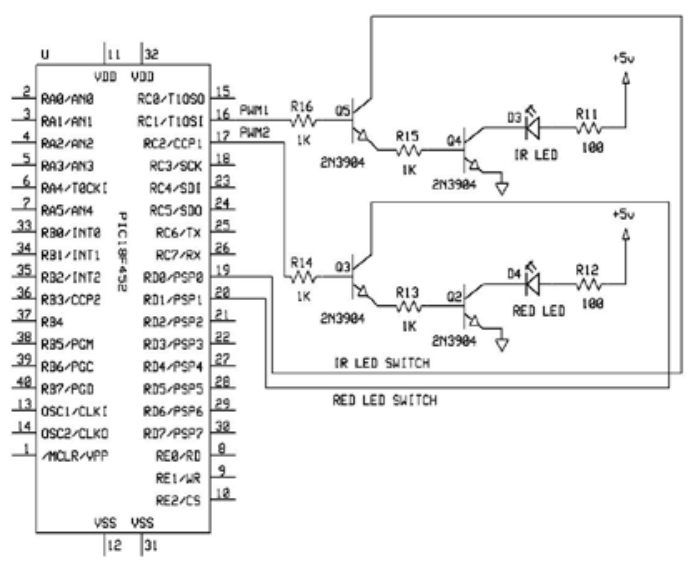

Figure 2: IR/RED LED Driver

\section{Methodologies}

\subsection{Hardware implementation}

The Pulse Oximeter hardware comprises the following components:

(1) IR and Red LED driver

(2) IR and Red LED light transmitter and light sensor photo (PIN) diode assembled in an ordinary plastic clothespin converted into a finger clip

(3) Light Source Amplifier

(4) Processor (Microchip PIC18F452)

(5) Display circuitry (LCD and RS232 interface with PC)

(6) Power Supply.

\subsubsection{IR and Red LED Driver}

Figure 2 shows the separate drivers that are used to drive IR and RED LED inside the finger clip. These drivers are made up of two pairs of 2N3904 NPN transistors in which the first transistors Q5 and Q3 operate as switches in order that the pulses come from pin 19 and pin 20 of the microcontroller PIC18F452 through collectors of the first transistors (Q5 and Q3) and to the base of the second transistors
Q4 and Q2. These transistors control the intensity of IR/RED LEDs by the Pulse Width Modulation PWM signals, which generated from pins 16 and 17 where assigned as PWM1 and PWM2 respectively. IR and RED LEDs are alternately switched every $150 \mu \mathrm{s}$ (micro second). The PWM1 and PWM2 signals are constructed in the software to regulate the intensity of IR and RED LEDs and are compensated for the ambient light variations.

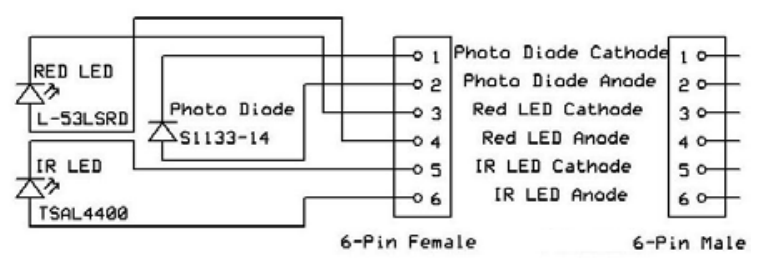

Figure 3: Finger Clip

\subsubsection{The Finger Clip}

The architecture of finger clip is demonstrated in Figure 3. The finger clip is made of an ordinary plastic clothespin and IR LED (TSAL4400) and RED LED (L-53LSRD) are resided on one appendage of the clip and are soldered on a small piece of printed circuit board. The photo sensor PIN diode (S1133-14) is soldered on a small piece of PCB to receive the transmitted light from IR LED and RED LED. The RED and IR LEDs are lit alternately every $150 \mu \mathrm{sec}$ (ON and OFF). There are six cables connected to the finger clip, and these cables are soldered to a 6-pin female header in order to connect with the main board through 6-pin male connector pins.

\subsubsection{Light Source Amplifier}

Figure 4 explains LM358 Operational Amplifier which is the main component of the trans-impedance amplifier to amplify very small amount of current (micro ampere) depending on the intensity of IR and visible RED light. The IR and RED light are sensed by the photo diode to $2 \mathrm{~V}$ to $3 \mathrm{~V}$ of analog voltage swing which is converted to digital numbers by the built-in ADC of embedded microcontroller PIC18LF452. In order to convert those digital numbers to $\mathrm{SPO}_{2}$ level in terms of percentage, the software program is implemented in the microcontroller which operates the mathematical routines. The signals are shown in 16 character 2 lines Hitachi HD44870 compatible LCD (figure 8). At the same time these data can be transmitted via USART (Universal Synchronous Asynchronous Receiver Transmitter) ports of the microcontroller to a PC. A Visual Basic 6 programming language is installed in the PC to display the wave forms, $\mathrm{SPO}_{2}$ level in percentage and pulse rate ... etc.

In this project, low power dual operational amplifier LM358 op-amp is chosen for its simplicity and 
routine. According to its data sheet, the operation from split power supplies is also possible and the low power supply current drain is independent of the magnitude of the power supply voltage. It consists of dual op-amps in a single package and it can operate at supply voltages as low as $3.0 \mathrm{~V}$ or as high as $32 \mathrm{~V}$ with low quiescent currents according to its data sheet (Appendix). Although one feature of LM358 is the maximum of $+32 \mathrm{~V}$, in this project the maximum voltage of $5 \mathrm{~V}$ is utilized. The single stage of this opamp can achieve voltage swings of 0VDC to common-collector voltage Vcc (pin 8) -1.5V [10], (appendix).

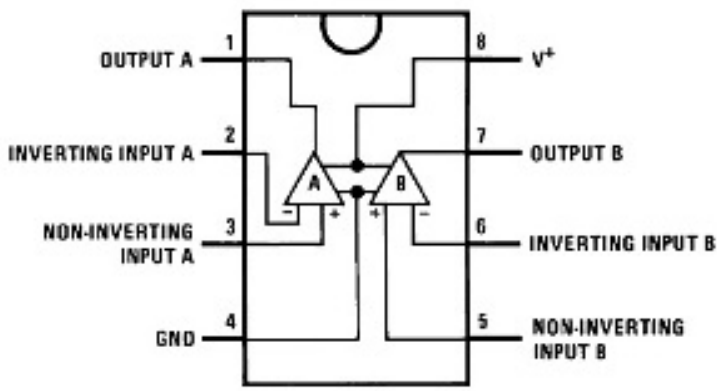

Figure 4: Low Power Dual Operational Amplifier LM358

By operating with $5 \mathrm{~V}$ power supply, the currents of 30mA pass through IR LED and RED LED each. The signal sensed by the Photo Diode D1 (Figure 5) passes through a high-pass filter formed by $\mathrm{C} 1$ and R2 that removes slow drift to non-inverting input of first op-amp (pin3). Figure 5 also explains that the high frequency noise is decoupled by a low-pass filter formed by $\mathrm{C} 2$ and R4 fed back from output of first op-amp (pin1) to inverting input of first op-amp (pin2). It amplifies the signal in the pass-band which is centered at $100 \mathrm{bpm}$ (beats per minute), by a factor of 100 .

The second stage of LM358 is configured as the previous stage in combination of filters and amplifier fabricated around second op-amp. The value of 500 is gained in the second stage. The overall gain of the two stages can be regulated with R5, 10K potentiometer. The output of first op-amp (pin1) in Figure 5 generates considerable amount of voltage with ripple at the peak of around $2.5 \mathrm{~V}$ which is sufficient enough to be converted by 10-bit ADC of the microcontroller PIC18LF452 through port RA0 configured as RANO (Analog Input) as shown in Figure 6. This amplified signal includes both DC and AC components representing infrared and visible red alternately.
The second stage output (pin7) is connected to a driver circuit to light the LED, indicating signal activity (Figure 5).

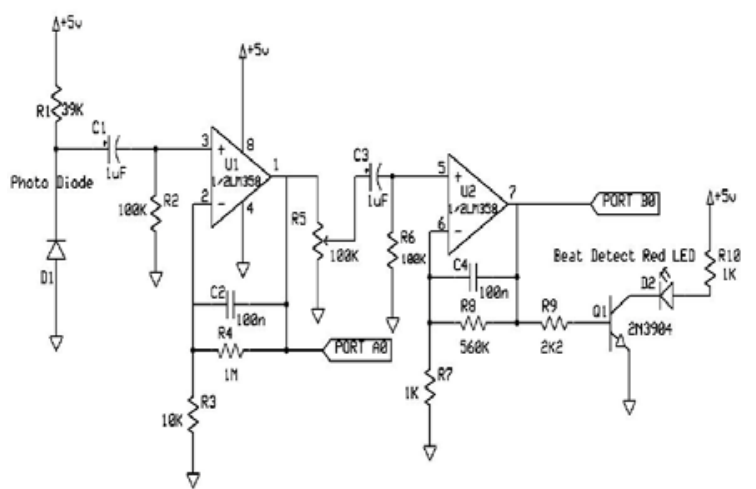

Figure 5: Light Source Amplifier

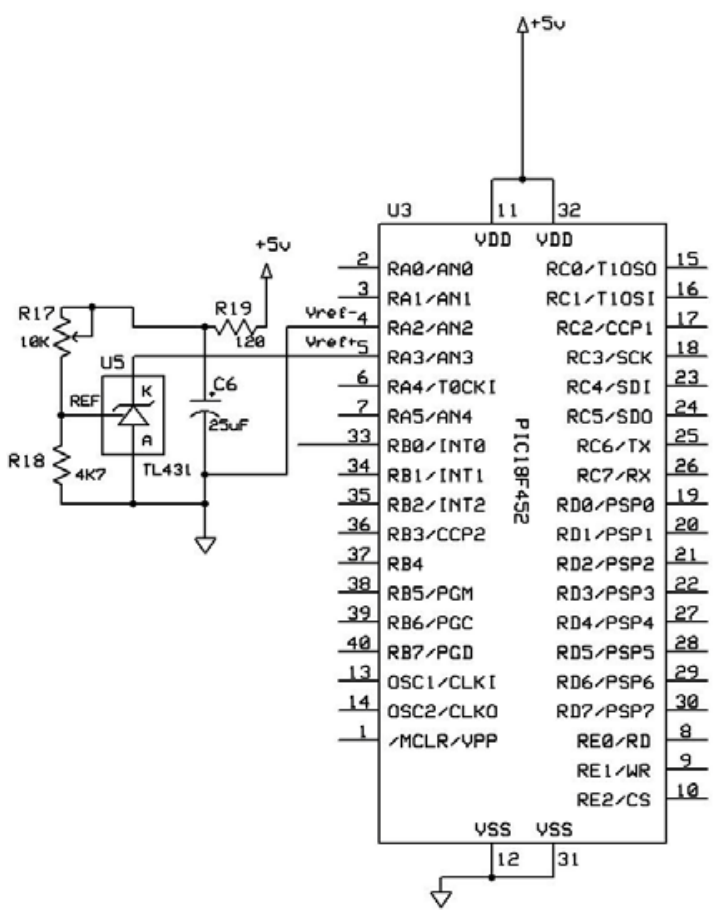

Figure 6: ADC Voltage Reference

\subsubsection{Processor (Microchip Microcontroller PIC18F452)}

The heart of the pulse oximeter is the Microchip PIC18F452-8bit microcontroller which has 8channel 10-bit ADC (Analogue to Digital Converter), 2 Capture/Compare/PWM CCP modules, 4 Timer/ Counter modules, Addressable USART (Universal Synchronous Asynchronous Receiver Transmitter module) and 3 External Interrupt pins among many peripheral features with 32 Kbytes of Flash Program Memory, 1536 bytes of RAM (Random Access Memory) and 256 bytes of EEPROM (Electrically Erasable and Programmable Read Only Memory) built on-chip. DC to $40 \mathrm{MHz}$ oscillator/clock input and $4 \mathrm{MHz}$ to $10 \mathrm{MHz}$ 
oscillator/clock input with PLL (Phase Lock Loop) which can speed up to 4 times of input frequency when PLL is active.

According to its data sheet, the synchronous serial port can be configured as either 3-wire Serial Peripheral Interface (SPI ${ }^{\mathrm{TM}}$ ) or the 2-wire InterIntegrated Circuit $\left(\mathrm{I}^{2} \mathrm{C}^{\mathrm{TM}}\right)$ bus and Addressable Universal Asynchronous Receiver Transmitter (AUSART) [11]. In this project, the microcontroller was programmed to switch and control the timing and intensity of IR and RED LEDs. Figure 6 explains that Pin 19 (RD0) and pin20 (RD1) are used for switching and pin 16 (RC1-PWM1) and pin17 (RC2-PWM2) are functioned for luminance changing values of Period and Duty Cycle in the software.

The ADC function (Figure 6) defines external Vref+ (Reference Voltage Positive) at pin 5 and Vref(Reference Voltage Negative) at pin 4. They are utilized as Voltage Reference instead of VDD (pin11-32) (Supply Voltage $+5 \mathrm{~V}$ ) to acquire an accurate reference voltage using TL431 Shunt Regulator and +3V (Vref + ). This is regulated by R17 (20K POT). VDD can be varied during system operation to obtain the accuracy of the ADC. Pin 4 (Vref-) is connected to Ground to set a Vref- to $0 \mathrm{~V}$.

\subsubsection{Display circuitry (LCD and RS232 interface with PC)}

The main display to show $\mathrm{SpO}_{2}$ concentration in percentage (\%) and Pulse Rate in BPM (beats per minute) is 16 character 2 line LCD JHD162A with back light (Hitachi HD44780 compatible) driven by Port B (RB2 to RB7) pin 35 to Pin 40 of microcontroller using 4-bit data (D4-D7) to save number of pins used, Figure 7. Although LED back light is available as the reflective light [12], LCD's back light is disabled to save power consumption in this project. The LCD panel is connected to the microcontroller via 9-pin connector.

Figure 8 shows the percentage of $\mathrm{SpO}_{2}$ and the heart beat rate of the author who is in good health.

Another type of display in this project is a PC (personal computer) with serial (USART) port and appropriate software installed. The microcontroller PIC18LF452 has built-in USART hardware on board at pins 25 and 26 (RC6/TX and RC7/RX), figure 7 that can send data to PC via RS-232 interface and display information such as $\mathrm{SPO}_{2}$ content in percent and/or Pulse Rate (beats per minute).

2N3904 transistors are used for the interface circuit instead of RS-232 chip for low- cost; it also saves the space for USART communication with PC, figure 9 .

\subsubsection{Power Supply}

The whole circuit is powered by regulate a $+5 \mathrm{~V}$, using LP2950 LDO (Low Drop-out Voltage Regulator) which can deliver up to $160-200 \mathrm{~mA}$ of current with constant $+5 \mathrm{~V}$. That is bipolar, low drop-out voltage regulators that can accommodate a wide input supply-voltage range of up to $30 \mathrm{~V}$ [13]. As the overall circuit consumes about $50-60 \mathrm{~mA}$, maximum current limit of $200 \mathrm{~mA}$ secures enough for even continuous operation. LP2950 is supplied to its input pin as low as $5.38 \mathrm{~V}$ DC at $100 \mathrm{~mA}$ to produce 5V regulated output to its LDO (Low Dropout) capability.

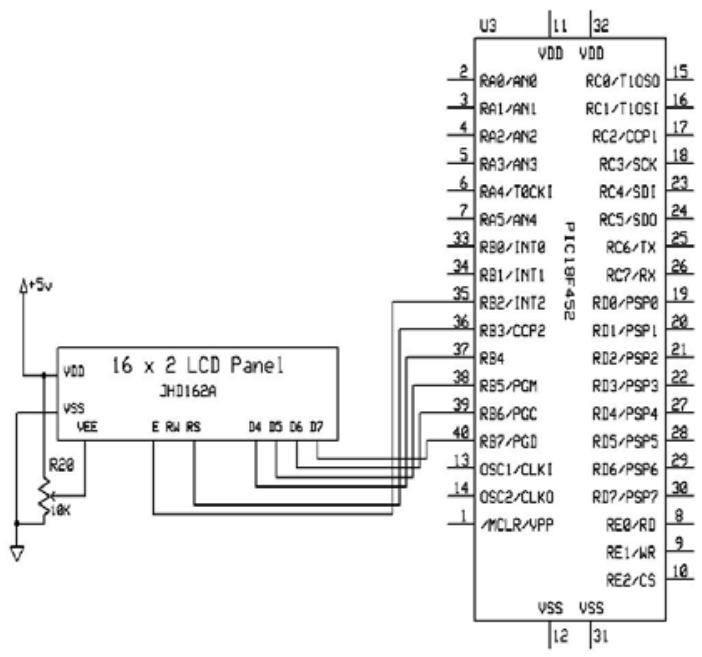

Figure 7: LCD Panel

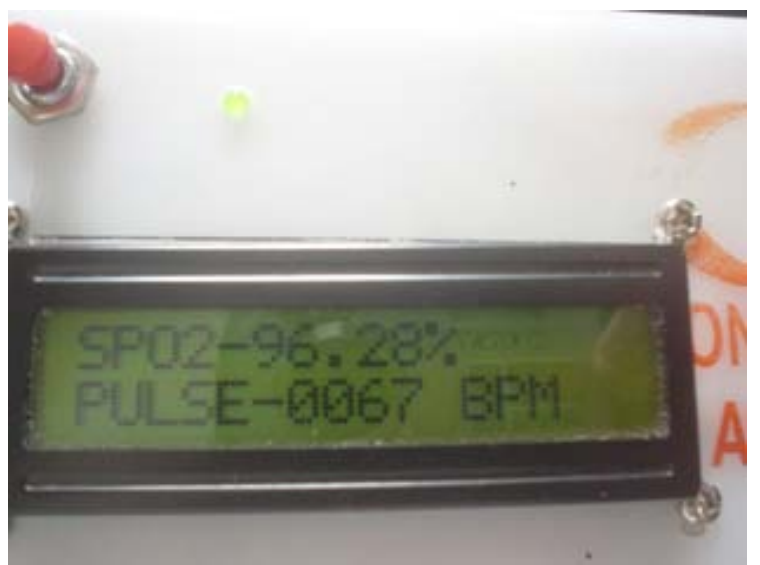

Figure 8: LCD display showing $\mathrm{SpO}_{2}$ and Pulse Rate

It is better to use batteries delivering more than $5.38 \mathrm{~V} \mathrm{DC}$ as the power source to prevent AC ripples and interference produced in most of the AC-DC adapters with poor filtering circuitry that can affect the accuracy of the pulse oximeter 


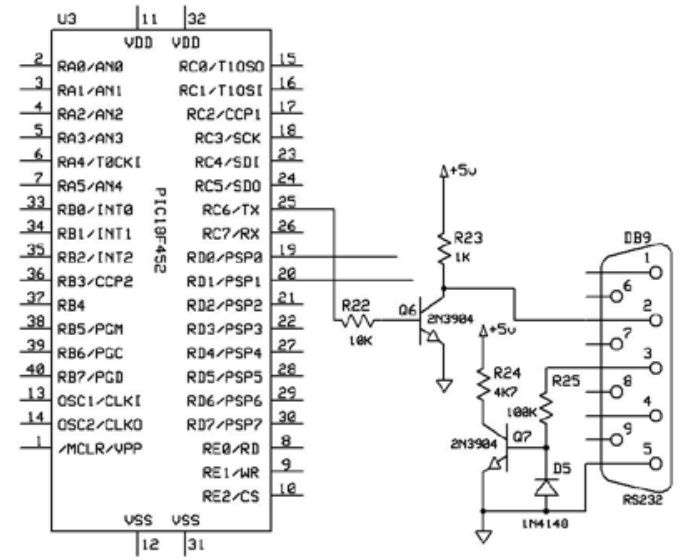

Figure 9: USART

\section{Analysis}

The software or firmware source code for the embedded system of pulse oximeter is written in Assembly language. It is compiled and programmed into PIC18LF452 Microcontroller using Microchip MPLAB IDE (Integrated Development Environment) that runs as a 32-bit application on MS Windows ${ }^{\circledR}$ [14] and PICkit2 programmer. MPLAB IDE is applied to implement the project, to simulate and debug the source code. The finalized source code is compiled to achieve the machine understandable HEX code and programmed or burnt into the microcontroller chip using PICkit2 programmer.

The features of the software program are expressed in the flow chart given in figure 10; they are depicted for clear visualization of the process in the software:

(1) Declaration of the Microcontroller,

(2) Configuration of the Microcontroller,

(3) RAM Assignment and Macros,

(4) Initialization and Setting of I/O (Input/Output) Ports,

(5) Setting up USART, ADC, CCP modules, Timer0, and Interrupt Controls,

(6) Wait and clear RAM space,

(7) Setting up LCD.

\subsection{MPLAB Program}

The main program executes the following order:

(1) Obtain sample readings of IR and RED without finger inside the finger clip, get the average of 10 readings then saved in assigned RAM space.

(2) Acquire the readings of IR and RED with finger inside the finger clip, get the average of 10 readings then saved in assigned RAM space.

(3) Calculate the Value $\mathrm{R}$ based on the readings using mathematical functions.

(4) Value R is multiplied by coefficient b, and then subtracted from coefficient a using mathematical functions $(S=a-b R)$. Store the value $S$ in the specified RAM to be shown as $\mathrm{SPO}_{2}$ level in percentage on LCD and/or PC.

(5) Only IR LED is lighted for more than 3 seconds to measure pulse rate BPM, and then calculated. The BPM value is stored in an assigned RAM for future display.

(6) Values of $\mathrm{SPO}_{2}$ (percent) and Pulse Rate (BPM) are displayed in LCD and/or PC.

(7) Repeat the above process from (1) to (6).

\section{Experimental Results}

Sample rate for IR and RED LED is $25 \mathrm{~ms}$ each which means IR and RED LEDs will be ON for 25 milliseconds. There is another sample rate for ADC (Analog to Digital Converter of microcontroller). It must be the same as IR and RED LEDs, i.e. $25 \mathrm{~ms}$ so that the sample data collection is synchronized. In practice (written in software), 20 samples are taken and only 10 samples are used but only one data, the averaged of the 10 data is saved in the RAM (Random Access Memory of microcontroller). It takes 100 ms to achieve $1 \times$ IR and $1 \times$ RED sample. Therefore, every 1 second we have collected, and saved in the RAM, 1 IR raw data and 1 RED raw data.

Switch ON the IR LED for 3 seconds and sense the pulsating signal due to blood flow by photodiode. The sensed electrical signal is amplified by LM358 and then sent to microcontroller interrupt pin RB0. B0 (Pin 33) (Figures 4,6,7,9) senses the rising edge of the AC signal (pulse signal) and counts the peaks and calculates the pulse rate in BPM and saves it in RAM. The result of $\mathrm{SPO}_{2}$ and PR (pulse rate) is updated on LCD and USART every 4 to 5 seconds, or exactly equals 4.194 seconds.

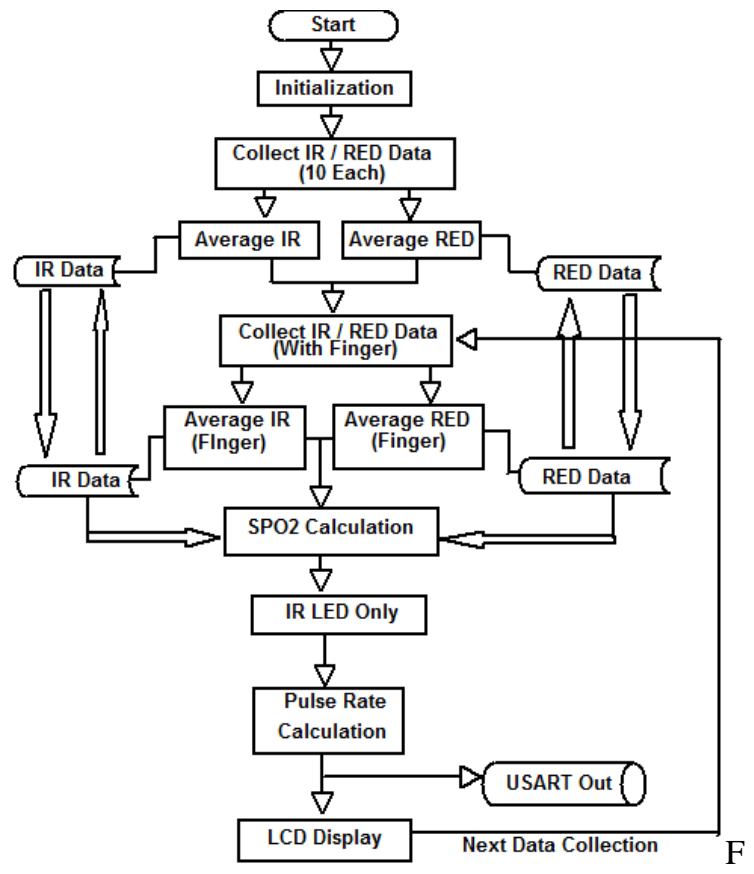

Figure 10: Flow Chart of Software 


\section{Conclusion}

The main objectives of this work are to develop a medical sensor node for real-time saturation of arterial oxygen, and heart beat rate monitoring using WSN technology. The design of pulse oximeter proposes the small size, light weight, low power consumption, standardized signal processing, data processing and wireless communication capabilities. The application of WSN technology into elderly healthcare anticipates supporting the healthcare professionals effectively and economically in longterm care conveniences.

\section{References}

[1] J.P. Lynch, "Overview of Wireless Sensors for the Real-Time Health Monitoring of Civil Structures," Proc. of the 4th International Workshop on Structural Control and Monitoring, New York, NY, June 10-11, 2004

[2] U. Anliker, et al., "AMON: A Wearable Multiparameter Medical Monitoring and Alert System”, IEEE Transactions on Information Technology in Biomedicine, 2004, 8(4)

[3] O. Marques, P. Chilamakuri, S. Bowser, J. Woodworth, "Wireless Multimedia Technologies for Assisted Living” Second LACCEI International Latin American and Caribbean Conference for Engineering and Technology (LACCEI’2004) "Challenges and Opportunities for Engineering Education, Research and Development” 2-4 June 2004, Miami, Florida, USA

[4] M. G.U. Segura, "Design of a wearable low power pulse oximeter for the nose", Chair for information technology with the emphasis communication electronics, UNIVERSITY OF ERLANGEN-NÜRNBERG, Erlangen, October 2008

[5] A.Young, "Wireless Oximeter" School of Information Technology and Electrical Engineering, University of Queensland, Submitted for the degree of Bachelor of Engineering (Honours) in the division of Computer Systems, October 2003

[6] T. Aoyagi, "Pulse oximetry: its invention, theory, and future," Journal of Anesthesia, 2003. pp. 259-66,

[7] P,B. Crilly, et al., "An Integrated Pulse Oximeter System for Telemedicine Applications," Proc. of the IEEE Instrumentation and Measurements Technical Conference, Ottawa, Canada, May 19-21, 1997

[8] M.B. Parlato, J.Meyer, B.Dzyubak, J.Helfenberger, "Low Cost Pulse Oximeter Probe”, In Conjunction with Engineering, World Health and the MEdCal Project, May 5,2009
[9] S.P. Dandamudi, "Introduction to Assembly Language Programming for Pentium and RISC processors”, ISBN 0387206361, November 2004, pp. 6-10

\section{Appendix}

LM358 Dual Differential Input Operational Amplifier Utilizing the circuit designs perfected for recently introduced Quad Operational Amplifiers, this dual operational amplifier features (1) low power drain (2) a common mode input voltage range extending to ground/VEE (3) Single Supply or Split Supply operation. The LM358 is equivalent to one half of an LM324.

This amplifier has several distinct advantages over standard operational amplifier types in single supply applications. It can operates at supply voltages as low as 3.0 Volts or as high as 32 Volts with low quiescent currents. The common mode input range includes the negative supply, thereby eliminating the necessity for external biasing components in many applications.

The output voltage range also includes the negative power supply voltage.

\section{Features}

1. Short circuit protected outputs

2. True differential input stage

3. Single supply operation: $3.0 \mathrm{~V}$ to 32 Volts

4. Low input bias currents

5. Internally compensated

6. Common mode range extends to negative supply

7. Single and split supply operation

8. ESD Clamps on the Inputs Increase Ruggedness of the Device without Affecting Operation
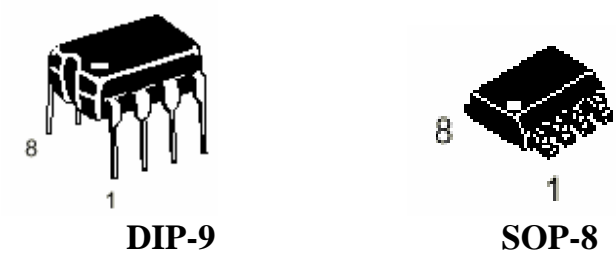

\section{ORDERING INFORMATION}

\begin{tabular}{|c|c|c|}
\hline Device & Temperature Range & Package \\
\hline LM358 & 0C to $+70^{\circ} \mathrm{C}$ & DIP- 8 \\
\hline LM358S & $0 \mathrm{C}$ to $+70^{\circ} \mathrm{C}$ & SOP- 8 \\
\hline
\end{tabular}

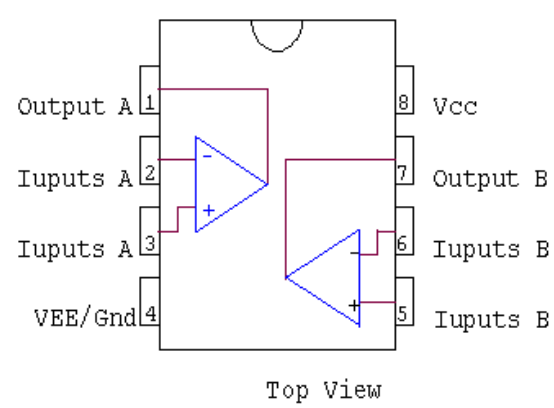

